\title{
BASES EPISTEMOLÓGICAS DE LAS ESTRATEGIAS DE TRIANGULACIÓN EN LA INVESTIGACIÓN SOCIOLÓGICA
}

\author{
Fernando Durán Pacheco*
}

La denominada "nueva filosofía de la ciencia", desde los años sesenta en adelante, ha procedido a una revisión sistemática de la historia de la ciencia para vincular el análisis epistemológico a la práctica real de la ciencia.

En términos generales, se reconoce que esta nueva perspectiva ofrece varias ventajas en relación a la visión histórica que presentaba la concepción clásica de la ciencia. Por una parte, la investigación sobre la historia de la ciencia deja de ser un hecho externo a la epistemología y se convierte en el marco desde el cual deben enfocarse los problemas epistemológicos. Además, la epistemología deja de ser vista con carácter normativo respecto a lo que debe ser el conocimiento científico, y la historia del quehacer investigador no tiene que adaptarse forzadamente a un modelo teórico que describe un curso lineal-acumulativo para esa actividad. Finalmente, se examinan desde una nueva óptica algunos antiguos problemas epistemológicos, enriqueciendo el examen de tales cuestiones.

Se ha destacado suficientemente el valor crítico de los planteamientos de esta corriente de pensamiento, pero parece existir cierta insatisfacción con respecto a la falta de las propuestas positivas que cabría esperar luego de un enjuiciamiento sistemático de las concepciones tradicionales ${ }^{1}$.

Uno de los exponentes de la nueva filosofía de la ciencia que parece representar un caso nítido de vigorosa crítica sin dar una respuesta constructiva igualmente catogórica a la necesidad de orientación que reclaman los investigadores, es Paul Feyerabend.

Ya en el primer trabajo que lo hizo conocido como uno de los nuevos filósofos de la ciencia, publicado en 1962, arremetió contra el dogmatismo en la investigación científica, que va circunscribiendo paulatinamente los métodos y conceptos por la incapacidad de imaginar alternativas al punto de vista que uno cree, y que tiene por consecuencia toda clase de maniobras para la conservación de ese punto de vista ${ }^{2}$.

En una serie de libros posteriores expuso la que él mismo denominó "una teoría anarquista del conocimiento".

Esa teoría parte de una aseveración muy radical: el método científico es una invención de los filósofos de la ciencia, pues no hay elementos que estén presentes en cada manifestación del quehacer de esas disciplinas y que expresen la existencia de tal método.

La práctica científica, según Feyerabend, es un proceso social de gran complejidad donde los especialistas, al tratar de resolver un problema, usan indistintamente los diferentes procedimientos adoptando sus métodos y modelos al problema que les ocupa, haciendo uso de todos los recursos que tienen a su alcance, incluso disparates.

\footnotetext{
* Académico Departamento de Sociología, Facultad de Ciencias Sociales Universidad de Chile

I J.A. Castorini y G.A. Palau, "Introducción", en Jean Piaget y Leo Apostel, Construcción y validación de las teorias cientificas, B. Aires: Ed. Paidós, 1986, especialmente pp.18-22.

${ }^{2}$ Paul Feyerabend, Límites de la ciencia (versión original, 1962) Barcelona: Paidós Ibérica, 1989, pp. 107-108.
} 
Este intrincado proceso es simplificado en forma arbitraria — podríamos añadir, inadmisible - por los filósofos de la ciencia, en una reconstrucción abstractamente elaborada que denominan método científico. Tal reconstrucción es un ejercicio de pensamiento racional que no tiene nada que ver con la práctica concreta antes mencionada. Pero ese método, entendido como si reflejara algo concreto, y sacralizado institucionalmente. tiene potencialidad para producir un estancamiento de la ciencia cuyos pasos quiere guiar pero a la cual tiende a quitar la flexibilidad que posee y que necesita para su cultivo.

Lá crítica a los filósofos tradicionales de la ciencia, que hace Feyerabend desde la perspectiva de una historia real de la práctica científica, quiere derribar los mitos que contiene la historia oficial que los filósofos han puesto en lugar de esa historia real. Entre enos mitos, la idea que la ciencia es una forma superior de conocimiento cuyos excelentes resultados se lograron gracias a la aplicación del método científico. Porque este autor considera que la ciencia ha obtenido preeminencia por su fuerza institucional (porque tiene instrumentos de poder tales como la educación formal, los expertos, las asociaciones profesionales) y no por la existencia y aplicación de reglas metodológicas estables que axpresen una supuesta racionalidad científica inmutable.

Vale la pena destacar en breve recuento los planteamientos que Feyerabend va desarrollando en sus obras y que se relacionan muy directamente con cuestiones de metodología de la investigación científica.

En su libro Contra el método, denominó "epistemología anarquista" a la idea que exicten modos de acercamiento diferentes como resultado de visiones distintas acerca de lo que queremos investigar, y que esos modos de acercamiento sólo pueden ser juzgados en cuanto apropiados o no apropiados según el punto de vista particular de quien realiza la investigación. Esta epistomología no sólo es preferible porque logra mejor conocimiento sino también porque es una opción más humana al ser coherente con la libertad del hombre ${ }^{3}$.

La idea de un método que contenga principios inalterables y absolutamente obligatorios para toda investigación no es confirmada por la historia de la ciencia. Más bien parece que los avances científicos importantes tuvieron lugar precisamente porque algunos investigadores decidieron no ligarse a esas reglas metodológicas o las violaron involuntariamente. Son las circunstancias de la investigación las que determinan la adopción de ciertos estándares, a veces opuestos a las reglas del llamado "método cientifico".

Creer en un método fijo y en una racionalidad fija supone una visión del hombre y de su contorno social demasiado ingenua. Sólo un principio metodológico general podría costenerse ante la complejidad de la tarea investigadora: todo vale ${ }^{5}$.

El trasfondo de este principio es el llamado a la afirmación de la libertad del científico, libertad que ha venido siendo restringida por la tradición, los formalismos y las creencias metafísicas. El principio "todo vale", cuya aplicación expresaría esa libertad en el terreno metodológico, significa el abandono de las reglas que abstractamente se han dado como definitorias de un método científico, y la apertura a un abanico amplio de

\footnotetext{
P. Foscrabend. Comtra el método (vorsión original, 1970), Barcelona: Ariel, 1974, pág. 13.

4 P. Fuerabend. Comura el método. pp. 15-17.

sp. Feycrabend. Comtra el método. pp. 21-22.
} 
métodos alternativos que puedan usarse según la naturaleza y necesidades que planteen los problemas que nos interesan ${ }^{6}$.

Feyerabend reclama una actitud nueva: la permanencia y estabilidad de un punto de vista no debe entenderse como signo de excelencia sino como indicador del fracaso de la razón para encontrar alternativas adecuadas que puedan utilizarse para trascender una etapa intermedia accidental de nuestro conocimiento. El pluralismo metodológico (y la proliferación de teorías) expresan esa nueva actitud que no se interesa por garantizar la estabilidad del conocimiento sino por vencer cualquier inmovilidad de la ciencia ${ }^{7}$.

Esa nueva actitud es posible si recordamos constantemente que las ciencias son nuestra propia creación, incluidos los severos estándares que parecen imponernos ${ }^{8}$.

Cinco años después, su obra Tratado contra el método, parte caracterizando a la ciencia como una empresa esencialmente anarquista y como proceso en el cual, por su naturaleza, el científico puede intervenir satisfactoriamente en la medida en que sea un oportunista 9 .

Pero el rechazo de los criterios universales y de las tradiciones rígidas no equivale a recomendar una nueva metodología. La intención de Feyerabend no es sustituir un conjunto de reglas metodológicas por otro conjunto, sino demostrar que todas las metodologías tienen limitaciones ${ }^{10}$.

Los científicos no resuelven problemas porque posean una metodología o una teoría de la racionalidad que funcione como varita mágica; mucho más importante es que han estudiado un problema por largo tiempo y conocen bien la situación. Si puede hablarse de método, éste sería el rechazo al conformismo y el aprovechamiento del poder de la imaginación, pues la ciencia requiere elasticidad para conseguir progreso; la metodología puede estrechar demasiado el campo del pensamiento ${ }^{11}$. Hay que hacer uso de todos los métodos, inclusive aquellos que podrían ser considerados acientíficos; y en ciertas ocasiones algunos procedimientos vistos tradicionalmente como científicos, podrían no usarse.

Estas ideas sobre el camino que sigue la ciencia realmente y en el cual debe insistirse se examinan con más detalle en los libros más polémicos que siguen al Tratado.

En La Ciencia en luna sociedad libre, afirma: "No hay ningún único procedimiento o conjunto de reglas que sea fundamental en toda investigación y garantice que es científica $y$, por consiguiente, digna de crédito. Todo proyecto, teoría o procedimiento ha de ser juzgado por sus propios méritos y de acuerdo con criterios que se adecuen al proceso en cuestión"12. No hay un método que sea medida invariable de adecuación; actualmente tenemos que hacer ciencia sin poder confiar en un método científico bien definido y estable. Pero la investigación no es arbitraria y carente de guía; los criterios existen, y derivan del propio proceso de investigación. no de concepciones abstractas de la racionalidad.

\footnotetext{
${ }^{6}$ La libertad también se manifiesta, en el plano térico. en un principio de prolferacion: intentar teorias que sean inconsistentes con el punto de vista aceptado. aunque este pusea una elevada confimacion: teorías que sean verdaderas

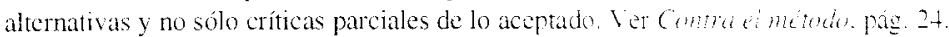

${ }^{7}$ P. Feyerabend. Comtra el métudo, pág. 30.

"P. Feyerabend. Comma el méterdo, pág. 134.

${ }^{9}$ P. Feyerabend. Tratado contra el método (version original, 1975 , Madrid: Tecnos, 1986, pág. 5.

${ }^{10}$ P. Feyerabend, Tratado contra el método, pág. 17

${ }^{11}$ P. Feyerabend, Tratado contra el método. pp. $297-298$

${ }^{12}$ P. Feyerabend, La ciencia en una sociedad libre (versión original. 1978). México: Siglo XXI, 1982, pp. 114-115.
} 
La investigación con éxito —-sostiene en Adiós a la razón- no obedece a estándares generales: se apoya a veces en una regla, a veces en otra, y no siempre se conocen explícitamente los movimientos que la hacen avanzar. Una teoría de la ciencia que señala normas comunes para todo tipo de indagación, no ayudaría al científico en su quehacer, pues no podría usarlas. Esa teoría de la ciencia es imposible; lo único que tenemos es el proceso concreto de investigación y reglas empíricas que pueden ayudarnos pero que tienen que ser siempre reexaminadas para asegurar que siguen siendo útiles, pues varían de un proyecto a otro ${ }^{13}$.

Más recientemente, en Diálogos sobre el conocimiento, el autor aclaró que su famoso principio "todo vale" no intentaba basar en el relativismo una nueva filosofía de la ciencia destinada a reemplazar los dogmas existentes (que no ayudan a realizar un trabajo científico); lo que quiere decir dicho principio es que debemos dejar a las ciencias hablar por sí mismas, teniendo en cuenta que su mensaje no puede resumirse en un sistema metodológico o en una teoría única. Lo más importante para el trabajo científico es sumergirse en el contexto de investigación adecuada ${ }^{14}$. Afirmar que todo vale, es decir que no hay reglas generales; no que cualquier cosa tienen igual utilidad para la investigación.

Podría sostenerse que en la base del pensamiento de Feyerabend hay supuestos que son compartidos por la mayoría de los filósofos de la ciencia ${ }^{15}$. Pero lo que parece definir mejor su contribución a la epistemología de la ciencia es la idea del pluralismo metodológico ${ }^{16}$.

Los debates en torno al "anarquismo", "relativismo" o "irracionalismo" de su posición, parecen de significación menor, y el propio Feyerabend se ha encargado de despejar los equívocos que tales etiquetas podrían suponer.

Lo positivo de su planteamiento es la afirmación que el ejercicio de la libertad en la ciencia consiste en reconocer y reforzar las tendencias pluralistas que esta actividad posee, tanto metodológica como teóricamente, estando siempre atentos a los resultados de investigaciones concretas o a los cambios prácticos, que en cualquier momento pueden reorientar nuestro trabajo de investigación. Las tradiciones de la ciencia no deben aceptarse a priori o descartarse igualmente, sino que deben ponderarse; en ningún caso el investigador debe cerrarse a los aportes que provienen de otros campos - del arte o la filosofía, por ejemplo- cuyas separaciones con la actividad científica son creaciones discutibles. En virtud de esos caracteres reales de la práctica científica, ésta ha producido descubrimientos y ha ampliado nuestros horizontes; por eso conviene guiarse por ellos.

Por más que esta posición puede ser vista como una formulación esencialmente crítica y demasiado vaga en sus recomendaciones como para servir en la orientación de la operación práctica de investigar, creemos que constituye una propuesta casi programática para enmarcar una estrategia metodológica que ha logrado gran desarrollo en la investigación sociológica actual. Nos referimos a la denominada triangulación.

Norman Denzin popularizó el nombre de triangulación para designar dicha estrategia metodológica ${ }^{17}$. Ella consiste, según la definición dada por Gerry Rose, en el uso de dos

\footnotetext{
${ }_{13}^{3}$ P. Feyerabend, Adiós a la razón (versión original, 1981), Madrid: Tecnos, 1984, pp. 20-22.

${ }_{14}$ P. Feyerabend, Diálogos sobre el conocimiento (versión original, 1991), Madrid: Cátedra, 1991, pp. 96-97

${ }^{15}$ Diego Ribes, Introducción a P. Feyerabend, Limites de la ciencia, op. cit., pág. 18.

16 Javier Echeverría, Introducción a la netodología de la ciencia, Barcelona: Barcanova, 1989, pp. 212-224.

17 Norman Denzin, The Research Act, Chicago: Aldine, 1970.
} 
o más diferentes métodos para estudiar el mismo fenómeno - puesto que ningún método es infalible, el uso de varios métodos daría resultados más concluyentes. La idea básica es que los datos obtenidos de un modo pueden contrastarse con los obtenidos de otra manera, apoyando el conjunto de la investigación ${ }^{18}$.

Denzin ha destacado que la utilización de una metodología triangulada en las Ciencias Sociales es apropiada sólo cuando nos parece que cada uno de los recursos que se combinan capturará ciertos elementos únicos en el proceso de investigación. La esencia de la triangulación es una percepción discriminante de lo que los investigadores consideran ser un mismo objeto.

Aplicar métodos disímiles puede ayudarnos enormemente en el estudio cuando se les enfoca sobre un mismo objeto; pero en cuanto cada uno de esos métodos es un modo diferente de reconstrucción de una realidad no se puede esperar una validación automática del conocimiento obtenido por esos distintos medios.

El empleo de métodos combinados no carece de antecedentes en la sociología. Algunas investigaciones consideradas clásicas en la disciplina se hicieron recurriendo a diversas técnicas de recolección y análisis de información. Puede recordarse que la denominada técnica de estudio-de-caso constituye una aproximación que utiliza procedimientos múltiples para estudiar la vida social de la comunidad. Tal es la óptica característica de los estudios realizados por Robert y Helen Lynd en Middletown, una típica comunidad estadounidense cuya estructura y procesos sociales fueron examinados usando distintos recursos metodológicos ${ }^{19}$.

Estudios-de-caso como los mencionados son precursores directos del actual enfoque de multimétodos. Este último, sin embargo, da un tratamiento más sistemático a los problemas relacionados con la combinación de varias técnicas dentro de un mismo diseño de investigación, y extiende el campo de aplicabilidad de este enfoque metodológico a un campo más amplio de situaciones que el limitado ámbito de la investigación sobre comunidades que constituyen el objeto de los clásicos estudios-de-caso.

Entre las técnicas de investigación más comunes en Sociología se cuentan el survey social, la experimentación, la observación-participante, la observación-no reactiva, y la investigación documental. Desde cierto punto de vista, cada una de esas técnicas produce información potencialmente válida acerca de la realidad; pero también puede decirse que, consideradas por sí solas, cada una de ellas no produce algunos tipos de información que pueden ser importantes o que los datos obtenidos tienen sesgos inherentes a las características de esa técnica particular.

Reconocer las debilidades de cada una de esas técnicas no es necesariamente aceptar que nuestra disciplina carece de capacidad para abordar empíricamente el estudio de la realidad social. Por el contrario, con la conciencia de esas debilidades se llega a la conclusión que tales técnicas no deben ser manejadas como alternativas mutuamente excluyentes entre las cuales debemos optar. Lo que ocurre es que esas debilidades no son idénticas, y al haber una diversidad de imperfecciones, podemos combinar las técnicas de modo que complementen sus fortalezas y al mismo tiempo compensen sus debilidades particulares.

\footnotetext{
${ }^{18}$ Gerry Rose, Deciphering Sociological Research, Londres: Mac-Millan, 1982, pp. 3-9 y 3-7.

${ }^{19}$ Robert y Helen Lynd, Middletown, New York: Harcourt, Brace, 1929; y Middletown in transition, New York: Harcourt, Brace, 1937.
} 
Sobre la base de estos supuestos, se estima que la utilización de métodos múltiples pueden ser una ayuda eficaz para el sociólogo en varias fases de la investigación, desde formular los problemas hasta el diseño de políticas fundadas en los resultados del estudio.

Pero la noción de triangulación se ha venido utilizando más específicamente en relación con la idea de medición múltiple. Es con respecto a la fase de medición en las investigaciones sociológicas donde se han explorado más detenidamente los problemas y ventajas de la estrategia de triangulación.

La medición, en términos amplios, consiste en asignar valores cuantitativos o cualitativos (expresables numéricamente, o en una distinción de tipos o clases) a las características o variables de una unidad que nos interesa estudiar. La medición triangulada trata de establecer esos valores viendo el fenómeno desde distintas perspectivas metodológicas.

Naturalmente, la triangulación supone que tiene sentido combinar recursos de medición cuando puede demostrarse empíricamente que, si tales recursos miden con error, esos errores se producen en aspectos diferentes. Por consiguiente, se requiere un cuidadoso análisis de cada recurso de medición en relación a los otros, y en relación al problema que se investiga 20 .

No se está hablando aquí de una validación convergente, en que el acuerdo entre las mediciones efectuadas por un medio y las mediciones realizadas por otro procedimiento nos permiten idealmente validar los resultados obtenidos por el primer instrumento. De lo que se trata es de lograr resultados distintos sin que esto signifique principalmente arrojar dudas sobre la validez de un instrumento, sino enriquecer la medición. La idea es que incluso técnicas que miden el mismo fenómeno, no entregarán resultados convergentes a menos que se corrijan los errores de medición propios de cada técnica. La triangulación, en ese caso, procura precisamente corregir tales errores utilizando la información que proviene de cada procedimiento de medición, perfeccionando con ello la validez de la operación total de medición.

Una clara ilustración puede encontrarse en el estudio empírico sobre frecuencia de los delitos de violación. Dada la resistencia de las víctimas a dar cuenta de la ocurrencia de esos delitos, los registros oficiales que contienen los casos reportados a la policía son considerados incompletos; en cambio, mayor fidelidad tienen las respuestas a entrevistas realizadas bajo el patrocinio de organismos de salud, aunque éstas tienen también la desventaja de obtenerse sólo para una muestra en tanto que los registros oficiales en el caso mencionado se refieren al universo. La medición triangulada aparece muy efectiva en una situación así, donde las deficiencias no se suman y las virtudes de los instrumentos se complementan.

Por el contrario, las estimaciones de frecuencia de infracciones de tránsito, tal como las registra la policía y como las declaran los causantes ante las entrevistas, adolecen de errores que van en similar dirección, puesto que hay selectividad de iguales consecuencias en los dos casos (por la cobertura parcial de los controles policiales y por la subestimación de los ciudadanos sobre la frecuencia con que cometen infracciones). En tal caso, la medición múltiple no cumple las finalidades que persigue la triangulación.

Aunque la triangulación se limite a la operación de medición, en sentido estricto,

\footnotetext{
${ }^{20}$ Un análisis de este tipo es efectuado por N.Denzin en "The methodologies of symbolic interaction", en Gregory Stone y Harvey Farheman, Social psychology thortugh symbolic interaction, New York: Xerox College Publishing, 1970
} 
utilizando múltiples instrumentos de medida en el contexto de una sola técnica matriz de investigación, el empleo de esa estrategia también afectará diversas etapas del estudio. La combinación de diversos instrumentos significa también recurrir a distintas fuentes de información y trabajar con varios co-investigadores, todo lo cual implica redefinir el tipo de trabajo que se realizaría si se aplicaran sólo los instrumentos convencionales que corresponden a la técnica matriz elegida.

Una de las ventajas más importantes de esa estrategia reside en que no solamente llama al conocimiento de las diversas técnicas disponibles para combinar, sino también en que estimula el desarrollo de nuevos instrumentos cuando se considera que los existentes introducen un tipo de error similar y no permiten la triangulación.

Otro aspecto importante de destacar se refiere a los modelos de medición. La conocida distinción entre lo que sería medir autoritarismo a nivel actitudinal o a nivel conductual, podría ilustrar este punto. Al emplear la triangulación es indispensable hacer explícitos los modelos de medición utilizados, puesto que al estudiar mediante diversos procedimientos un mismo fenómeno, los resultados diferentes pueden deberse a esos modelos de medición distintos y no a errores de medición. Cuando se utiliza un solo procedimiento, no hay habitualmente una especial preocupación por explicitar esos modelos de medición; pero dentro de una estrategia de triangulación, es importante detectar si los errores se deben a la medición de diferentes conceptos o a la aplicación de distintos modelos de medición para medir un mismo concepto. Cuando los resultados de las mediciones difieren, tendemos a pensar que nuestros instrumentos miden algo bastante diferente; pero a la vez la noción teórica que queremos medir en ambos casos es la misma y en tal caso se debe esclarecer qué modelos de medición están involucrados. Así como pueden emplearse diferentes instrumentos para medir un mismo constructo teórico, en una operación de triangulación, a veces hay distintos modelos de medición referidos a ese constructo y es preciso distinguir ambas cuestiones.

La noción de medición triangulada parece muy fructífera para manejar las relaciones entre las técnicas cuantitativas y cualitativas, que tradicionamente han sido objeto de cierto enfrentamiento. Quizás esto último no haya sido del todo dañino, puesto que su respectivo desarrollo relativamente independiente se dice que ha generado un depósito de experiencia diferente, de modo que su empleo conjunto podría permitir el descubrimiento de fuentes de sesgos y de medios para manejarlos que han permanecido ignorados en las dos tradiciones aisladas ${ }^{21}$.

El planteamiento de estrategias de triangulación como un recurso metodológico, encuentra apoyo en la concepción epistemológica elaborada por Feyerabend. La legitimidad del empleo de diferentes medios para abordar un problema y el estímulo a poner en juego la imaginación para ponderar la mejor manera de utilizar las tradiciones más valederas de la disciplina en la solución de las dificultades actuales de la investigación, coinciden con la búsqueda de formas creativas de combinación de instrumentos de medida que requiere la triangulación.

A su vez, la propuesta de Feyerabend parece encontrar una aplicación específica en dicha estrategia metodológica, en la que puede proyectarse su potencialidad orientadora.

${ }^{21}$ Charles Reichardt y Thomas Cook, "Hacia una superación del enfretamiento entre métodos cualitativos y cuantitativos", en Th. Cook y Ch. Reichardt. Métodos cuantitatives y cualitativos en investigación evaluativa, Madrid, 1988, pág. 47. 
En la triangulación hay un modo concreto en el cual las reglas empíricas que fluyen de la historia de la práctica científica pueden canalizar una completación de perspectivas que no se maneja aleatoriamente sino con rigurosa preocupación por adicionar las ventajas de enfoques diferentes.

La Sociología ha mostrado especial receptividad a la complementación de lo que hoy se denomina "estilos de investigación". La corriente que sostiene una propuesta de métodos múltiples ${ }^{22}$ parece ganar terreno entre los investigadores; con esto también crece el interés por afinar los problemas técnicos — como los de la medición triangulada—que envuelve la operación conjunta de métodos disímiles. Para ese fin, concepciones epistemológicas como la que sostiene Feyerabend pueden resultar especialmente iluminadoras.

22 John Brewer y Albert Hunter, Multimethod Research: A Synthesis of Styles, California: sage, 1989, pp. 22-25. 